\title{
Tendencia en el estudiante de medicina a ejercer como médico general o especialista
}

\author{
Hayo Breinbauer $K^{1 a}$, Germán Fromm $R^{a}$, Daniela Fleck $L^{b}$, \\ Luis Araya $C^{1 a}$.

\section{Trends among medical students towards general practice or specialization}

Background: A 60/40 ratio has been estimated as a country's ideal proportion between general practiotioners and specialists. In Chile this proportion was 36/ 64 in 2004, exactly the opposite of the ideal. Trends towards specialization or general practice among medical students have not been thoughfully studied. Aim: To assess trends among medical students towards becoming general practitioners or specialists, exploring associated factors. Ma terial and methods: Descriptive survey of 822 first to seventh year medical students at the University of Chile, School of Medicine. Desired activity to pursue (general practice or specialization) after graduation and general orientations within clinical practice were explored. Results: Fifty three percent of students desired to enter a specialization program. Only $20 \%$ would work as a general practitioner (27\% were still indecisive). Furthermore, a trend in early years of medical training towards an integral medicine is gradually reversed within later years. Seventh year students give significantly more importance to specialization than to integral medicine $(p<0.01)$. Ten percent of this opinion change is related to the emphasis given to specialized medicine in the teaching environment. Conclusions: Most students prefer to enter a specialization program immediately after finishing medical school. Moreover, there is a social trend, at least within the teacher-attending environment, promoting not only the desire to specialize, but a pro-specialist culture (Rev Méd Chile 2009; 137: 865-72). (Key w ords: Education, medical, undergraduate; Primary health care; Specialties, medical)

Recibido el 11 de abril, 2008. Aceptado el 12 de mayo, 2009.

Este trabajo no recibió ayuda financiera. El total de los costos de su implementación fueron aportados por fondos personales de los investigadores.

${ }^{1}$ Unidad de Bioética, Facultad de Medicina, Campus Occidente, Universidad de Chile.

aPsicólogo

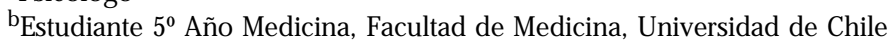

$\mathrm{E}^{1}$ continuo avance de las ciencias biomédicas hace imposible para un único individuo aunar la totalidad del conocimiento médico, convirtiendo al especialista, y más aún al subespecialista, en

Correspondencia a: Dr. Hayo Breinbauer K. Walter Scott 1439, Vitacura. Teléfono celular: 8-2284576. Teléfono fijo: 4948861. E mail: hayo.bk@gmail.com una necesidad irrefutable de la práctica médica ${ }^{1,2}$. Experiencias en países desarrollados con sistemas de salud bien organizados, señalan que las necesidades poblacionales estarían bien cubiertas cuando la proporción entre médicos generales (MG) y médicos especialistas (ME) se acerca a 60/403. En Chile, en los años en torno a 1950 esta proporción se estimaba en 70/30 experimentando grandes 
cambios en las últimas décadas, invirtiéndose a favor del ME. La relación MG/ME se estimaba en $44 / 56$ en $1996^{4}$ y 36/64 en $2004^{3}$, situación contraria al ideal propuesto. Si se analiza al sector público sin considerar municipalidades, esta cifra aumenta a $13 / 84^{3}$.

En términos absolutos, se estima que aunque es necesario contratar 888 especialistas con cargos de 44 horas semanales para suplir las necesidades del régimen de Garantías Explícitas en Salud (GES), se necesitarían 1.235 médicos generales para satisfacer la demanda asistencial en atención primaria en el país 5 .

Más aún, la tendencia del MG es a transformarse rápidamente en $\mathrm{ME}$, demostrando una altísima rotación: 40\% de los MG alcanza menos de 1 año de antigüedad y $69 \%$ no supera los 3 años ${ }^{6}$.

Este aumento en la proporción del $\mathrm{ME}$ en desmedro del MG responde a un complejo fenómeno social, que evidencia una tendencia general hacia la especialización. Dentro de sus diversos factores se han esbozado: 1) la falta de proyección económica, de perfeccionamiento y desarrollo científico para el MG, especialmente en el sector público; 2) la mayor presencia de tecnología y poder resolutivo en el campo del ME; 3) una influencia en la educación médica universitaria con tendencia al $\mathrm{ME}$; entre otras $^{5,6}$. La objetivación de estas inferencias ha sido escasa en nuestro medio, así como su impacto en el interés del médico recién egresado por elegir desempeñarse como MG. Un estudio cualitativo en 38 estudiantes (8 de los cuales cursaban el último año de la carrera) determinó mediante grupos focales que $60 \%$ de los alumnos estaría dispuesto a ejercer como MG ${ }^{7}$. Fuera de esta experiencia, no se ha evaluado la proyección al egresar de los estudiantes en términos de MG o ME, ni de factores asociados.

Más allá del decidir ejercer como MG o ME, se ha planteado la existencia de una actitud "predominantemente especialista" dentro de la práctica clínica cotidiana. Esta tendencia premiaría una alta resolutividad parcelada por campo de una especialidad, en desmedro de la visión global de la salud del individuo ${ }^{6,8-10}$.

Aunque un ME puede efectivamente presentar una actitud integral considerando al paciente en su contexto bio-psico-social, la opinión informal considera esta situación como la excepción, siendo la regla una atención fragmentada por especialidades ${ }^{2,11,12}$.
Buscando evaluar estos aspectos, los objetivos del presente estudio fueron: 1) objetivar la intención de los estudiantes de medicina a desempeñarse como MG o ME al egresar, 2) explorar la tendencia, tanto de estudiantes como del medio universitario donde se desenvuelven, hacia una práctica médica orientada a la especialidad o hacia la medicina general-integral.

\section{MATERIAL Y MÉTODO}

Estudio descriptivo-transversal, realizado entre mayo y octubre de 2007. El universo consideró el total de 1.438 alumnos cursando entre primer y séptimo año de la carrera de medicina durante 2007, en la Universidad de Chile. Para cada año de la carrera, se eligió una instancia de asistencia masiva (como clases magistrales), durante la cual se encuestó a los alumnos asistentes. La muestra reunida incluyó 822 estudiantes, representando $57,2 \%$ del universo. La proporción de estudiantes de tercer año que participaron en la muestra (con respecto al universo) fue significativamente más alto que el promedio y que cualquier otro curso ( $72,1 \%$ vs $57,2 \% \mathrm{p} \varangle 0,05)$. No hubo otras diferencias significativas en el porcentaje de participantes entre los cursos ( $p<0,05$ ver Tabla 1 ). Se eliminaron 23 cuestionarios por tener respuestas en blanco o ilegibles para los indicadores analizados, dejando un " $n$ " válido de 799 estudiantes.

Previo consentimiento informado, se aplicó un instrumento diseñado en base a una encuesta cualitativa previa a 31 estudiantes. Mediante indicadores tipo Lickert se indagó sobre actitudes y preferencias de los estudiantes frente a ejercer como MG o ME (Tabla 2). Se prefinó esta metodología cuantitativa buscando evaluar un mayor número de estudiantes (al requerir menor tiempo de aplicación del instrumento), obtener resultados concretos sobre la proporción de estudiantes con una u otra tendencia, y poder comparar los distintos cursos entre sí. Determinar causas subyacentes a estas tendencias requerirá en el futuro de estudios cualitativos en profundidad.

Los resultados obtenidos muestran alta concordancia con una aplicación previa del instrumento (7 meses antes, en 149 estudiantes del campus occidente de la facultad ${ }^{13}$ ), apoyando la confiabilidad del instrumento (índice kappa ponderado 0,67 a 0,89 para indicadores comparables, entre ambas aplicaciones).

Se realizaron pruebas de comparación de proporciones (prueba z), de comparación de prome- 
Tabla 1. Muestra del estudio

\begin{tabular}{|lllllllll|}
\hline M uestra de estudiantes & & & & & & & \\
& $\mathbf{1}^{\text {er }}$ año & $\mathbf{2}^{\mathbf{0}}$ año & $\mathbf{3}^{\text {er }}$ año & $\mathbf{4}^{\mathbf{0}}$ año & $\mathbf{5}^{\mathbf{0}}$ año & $\mathbf{6}^{\mathbf{0}}$ año & $\mathbf{7 0}^{\mathbf{0}}$ año & Total \\
\hline Estudiantes matriculados* & 217 & 226 & 201 & 184 & 189 & 210 & 211 & 1.438 \\
Estudiantes que & 129 & 139 & 145 & 91 & 94 & 116 & 108 & 822 \\
respondieron la encuesta & $(59,4 \%)$ & $(61,5 \%)$ & $(72,1 \%)^{* *}$ & $(49,4 \%)$ & $(49,7 \%)$ & $(55,2 \%)$ & $(51,2 \%)$ & $(57,2 \%)$ \\
Número de encuestas & 127 & 138 & 143 & 87 & 84 & 115 & 105 & 799 \\
con respuestas válidas & $(58,5 \%)$ & $(61,1 \%)$ & $(71,1 \%)^{* *}$ & $(47,3 \%)$ & $(44,4 \%)$ & $(54,8 \%)$ & $(49,8 \%)$ & $(56,6 \%)$ \\
\hline
\end{tabular}

*En el año 2007. **El grupo que respondió la encuesta de tercer año resultó ser significativamente mayor a los otros cursos y al promedio de los estudiantes ( $p<0,05$ en prueba $z$ ).

Se describen el número de alumnos matriculados en cada año de la carrera y la proporción de éstos que fueron encuestados. Se describe además los cuestionarios válidos utilizados en el análisis. La muestra de tercer año resultó ser significativamente mayor a todos los otros cursos.

Tabla 2. D escripción de los indicadores en la Encuesta

\section{Indicadores incluidos en la Encuesta}

1. Después de $7^{0}$ año, le gustaría:

a. Ejercer como Médico General.

b. Ingresar a un programa de formación de Especialidad. Indique qué Especialidad elegiría:

c. No sé.

2. ¿En su práctica cotidiana como futuro médico, qué importancia le da a? (en un puntaje de 1 a 10 siendo 1 lo mínimo y 10 lo máximo)

a. Resolver el/los problema(s) de salud, que me corresponda según mi especialidad y cargo.

b. Atención y solución integral, de los problemas de salud de un paciente en su totalidad.

3. ¿Con qué énfasis se promueven los siguientes aspectos, en el contexto en el cual usted se desenvuelve (hospital, consultorio, universidad)? (en un puntaje de 1 a 10 siendo 1 lo mínimo y 10 lo máximo)

a. Resolver el/los problema(s) de salud, que me corresponda según mi especialidad y cargo.

b. Atención y solución integral, de los problemas de salud de un paciente en su totalidad.

Para efectos de la interpretación de los resultados, se detallan textualmente las preguntas incluidas en el instrumento. Este fue aplicado a 822 estudiantes de primer a séptimo año de la carrera de medicina, obteniéndose 799 respuestas válidas.

dios (prueba t de Student) y correlaciones parciales entre las variables, corrigiendo por año de la carrera, utilizando software estadístico SPSS 16.0.

\section{RESULTADOS}

Al terminar la carrera, la mayonía de los estudiantes (53,2\%) preferińan formarse como ME, mientras que sólo 19,9\% declara querer ejercer como MG (Tabla
3). El 26,9\% restante declara no saber qué hará al egresar. Esta tendencia es mayor en primer año (58,3\% hacia ME) y desciende significativamente hacia quinto año $(45,2 \% ; \mathrm{p}<0,05)$. Durante el internado se aprecia un nuevo ascenso, donde $51,4 \%$ de los médicos a punto de egresar declara querer ingresar a un programa de especialidad.

Destaca que 10,4\% de estudiantes que declaran querer especializarse, sin saber en cuál especialidad. Esta tendencia se vislumbra desde primer año. 
Tabla 3. Preferencias al egresar

\begin{tabular}{|c|c|c|c|c|c|c|c|c|}
\hline \multicolumn{9}{|c|}{ Preferencias por año de la carrera, frente a la actividad a realizar al egresar } \\
\hline $\begin{array}{l}\text { Número de estudiantes } \\
\text { por año de la carrera* }\end{array}$ & $\begin{array}{l}1^{\mathrm{er}} \text { año } \\
127 \\
(100 \%)\end{array}$ & $\begin{array}{l}2^{0} \text { año } \\
138 \\
(100 \%)\end{array}$ & $\begin{array}{l}3^{e r} \text { año } \\
143 \\
(100 \%)\end{array}$ & $\begin{array}{l}4^{0} \text { año } \\
87 \\
(100 \%)\end{array}$ & $\begin{array}{l}5^{0} \text { año } \\
84 \\
(100 \%)\end{array}$ & $\begin{array}{l}60 \text { año } \\
115 \\
(100 \%)\end{array}$ & $\begin{array}{l}70 \text { año } \\
105 \\
(100 \%)\end{array}$ & $\begin{array}{l}\text { Total } \\
799 \\
(100 \%)\end{array}$ \\
\hline $\begin{array}{ll}\text { *. } & \text { Formación como } \\
\text { 2: } \\
\text { o․ }\end{array}$ & $\begin{array}{l}74 \\
(58,3 \%)\end{array}$ & $\begin{array}{l}81 \\
(58,7 \%)\end{array}$ & $\begin{array}{l}82 \\
(57,3)\end{array}$ & $\begin{array}{l}36 \\
(41,4 \%)\end{array}$ & $\begin{array}{l}38 \\
(45,2 \%)\end{array}$ & $\begin{array}{l}60 \\
(52,2 \%)\end{array}$ & $\begin{array}{l}54 \\
(51,4 \%)\end{array}$ & $\begin{array}{l}425 \\
(53,2 \%)\end{array}$ \\
\hline 过 Médico General & $\begin{array}{l}19 \\
(15 \%)\end{array}$ & $\begin{array}{l}22 \\
(15,9 \%)\end{array}$ & $\begin{array}{l}24 \\
(16,8)\end{array}$ & $\begin{array}{l}21 \\
(24,1 \%)\end{array}$ & $\begin{array}{l}23 \\
(27,4 \%)\end{array}$ & $\begin{array}{l}25 \\
(21,7 \%)\end{array}$ & $\begin{array}{l}25 \\
(23,8 \%)\end{array}$ & $\begin{array}{l}159 \\
(19,9 \%)\end{array}$ \\
\hline ๑ No sé & $\begin{array}{l}34 \\
(26,8 \%)\end{array}$ & $\begin{array}{l}35 \\
(25,4 \%)\end{array}$ & $\begin{array}{l}37 \\
(25,9 \%)\end{array}$ & $\begin{array}{l}30 \\
(34,5 \%)\end{array}$ & $\begin{array}{l}21 \\
(25 \%)\end{array}$ & $\begin{array}{l}30 \\
(26,4 \%)\end{array}$ & $\begin{array}{l}26 \\
(24,8 \%)\end{array}$ & $\begin{array}{l}212 \\
(26,5 \%)\end{array}$ \\
\hline $\begin{array}{l}\text { Estudiantes que saben qué } \\
\text { especialidad escogerían }\end{array}$ & $\begin{array}{l}53 \\
(41,7 \%)\end{array}$ & $\begin{array}{l}66 \\
(47,8 \%)\end{array}$ & $\begin{array}{l}58 \\
(40,6 \%)\end{array}$ & $\begin{array}{l}26 \\
(29,9 \%)\end{array}$ & $\begin{array}{l}32 \\
(38,1 \%)\end{array}$ & $\begin{array}{l}56 \\
(48,7 \%)\end{array}$ & $\begin{array}{l}51 \\
(48,6 \%)\end{array}$ & $\begin{array}{l}342 \\
(42,8 \%)\end{array}$ \\
\hline $\begin{array}{l}\text { Estudiantes que quieren } \\
\text { especializarse, pero no saben } \\
\text { qué especialidad elegir }\end{array}$ & $\begin{array}{l}21 \\
(16,5 \%)\end{array}$ & $\begin{array}{l}15 \\
(10,8 \%)\end{array}$ & $\begin{array}{l}24 \\
(16,8 \%)\end{array}$ & $\begin{array}{l}10 \\
(11,5 \%)\end{array}$ & $\begin{array}{l}6 \\
(7,1 \%)\end{array}$ & $\begin{array}{l}4 \\
(3,5 \%)\end{array}$ & $\begin{array}{l}3 \\
(2,8 \%)\end{array}$ & $\begin{array}{l}83 \\
(10,4 \%)\end{array}$ \\
\hline
\end{tabular}

*Considerando sólo respuestas válidas.

Se presentan los resultados de la encuesta ordenados por año de la carrera. Se describe el número de estudiantes que marcaron cada opción y el porcentaje relativo al número de estudiantes totales por cada año. Destaca la elección de formarse como especialista como la primera opción para la mayonía de los estudiantes.

Durante los primeros 4 años de la camera, la gran mayonía de los estudiantes que declara querer especializarse, eleginía medicina intema (Tabla 4). En sexto año ocupan esta preferencia psiquiatría y pediatría, y en séptimo año cirugía y ginecología-obstetricia. En este último año, se aprecia una distribución más homogénea por las distintas altemativas.

Más allá de la decisión explícita por ser MG o $\mathrm{ME}$ al egresar, durante los primeros años los estudiantes asignan significativamente más importancia a practicar una atención de orientación integral del paciente por sobre una centrada en resolver exclusivamente los problemas atingentes a una especialidad ( $p<0,01$; Figura 1).

Sin embargo, esta tendencia se invierte durante el internado: cobra mayor importancia una práctica orientada a la "atención por especialidades", superando significativamente la relevancia asignada a una "atención integral" ( $p \varangle 0,01$ ). En cuanto a la percepción sobre el énfasis con que promueven estos aspectos, en primer año el alumno percibe el entomo donde se desempeña como primordialmente promo- tor de una "atención integral" ( $p \varangle 0,01$; Figura 2). Sin embargo, esto disminuye drásticamente hacia años posteriores, alcanzando valores mínimos en nuestro instrumento. Paralelamente, el énfasis con que se promueve la atención por especialidades se incrementa gradualmente. Desde cuarto año en adelante, el énfasis pro-especialista es significativamente mayor ( $p \varangle 0,01$ ) a la promoción pro-integral.

Conceptualmente es razonable pensar, que el énfasis con que los estudiantes perciben que uno de estos aspectos es promovido, influye sobre la importancia que ellos mismos asignan a dicho aspecto. El análisis de correlaciones parciales permite evaluar el peso que tiene una variable sobre la otra, al comparar la correlación existente entre los cambios que presenta cada variable, entre los distintos años de la carrera.

Desde este enfoque, podemos inferir que 12,3\% del cambio observado en la importancia que estudiantes asignan a una "atención integral", podnía ser explicada por el descenso que tiene la promoción de este aspecto en su contexto social $(r=0,123 ; p \varangle 0,05)$. 
Tabla 4. Especialidades deseadas

\begin{tabular}{|c|c|c|c|c|c|c|c|}
\hline $\begin{array}{l}\text { Especialidad } \\
1^{\text {er }} \text { año } \\
53 \text { alumnos }\end{array}$ & $\begin{array}{l}\text { es más elegidas } \\
2^{0} \text { año } \\
66 \text { alumnos }\end{array}$ & $\begin{array}{l}\text { por año de la } \\
3^{\text {er año }} \\
58 \text { alumnos }\end{array}$ & $\begin{array}{l}\text { arrera } \\
40 \text { año } \\
26 \text { alumnos }\end{array}$ & $\begin{array}{l}5^{0} \text { año } \\
32 \text { alumnos }\end{array}$ & $\begin{array}{l}6^{0} \text { año } \\
56 \text { internos }\end{array}$ & $\begin{array}{l}7^{0} \text { año } \\
51 \text { internos }\end{array}$ & $\begin{array}{l}\text { Total } \\
342 \text { alumnos }\end{array}$ \\
\hline $\begin{array}{l}\text { Medicina } \\
\text { Intema } \\
(30,2 \%)\end{array}$ & $\begin{array}{l}\text { Medicina } \\
\text { Intema } \\
(42,4 \%)\end{array}$ & $\begin{array}{l}\text { Medicina } \\
\text { Intema } \\
(44,8 \%)\end{array}$ & $\begin{array}{l}\text { Medicina } \\
\text { Interna } \\
(50 \%)\end{array}$ & $\begin{array}{l}\text { Cirugía } \\
(34,3 \%)\end{array}$ & $\begin{array}{l}\text { Psiquiatńa } \\
(16,1 \%)\end{array}$ & $\begin{array}{l}\text { Cinugía } \\
(17,6 \%)\end{array}$ & $\begin{array}{l}\text { Medicina } \\
\text { Intema } \\
(12,8 \%)\end{array}$ \\
\hline $\begin{array}{l}\text { Cinugía } \\
(20,8 \%)\end{array}$ & $\begin{array}{l}\text { Cirugía } \\
(25,8 \%)\end{array}$ & $\begin{array}{l}\text { Cirugía } \\
(29,3 \%)\end{array}$ & $\begin{array}{l}\text { Cirugía } \\
(30,7 \%)\end{array}$ & $\begin{array}{l}\text { Medicina } \\
\text { Interna } \\
(25 \%)\end{array}$ & $\begin{array}{l}\text { Pediatría } \\
(14,3 \%)\end{array}$ & $\begin{array}{l}\text { Ginecología- } \\
\text { Obstetricia } \\
(15,7 \%)\end{array}$ & Cinugía \\
\hline $\begin{array}{l}\text { Pediatría } \\
(15,1 \%)\end{array}$ & $\begin{array}{l}\text { Pediatŕa } \\
(7,6 \%)\end{array}$ & $\begin{array}{l}\text { Pediatría } \\
(10,3 \%)\end{array}$ & $\begin{array}{l}\text { Fisiatría } \\
(7,6 \%)\end{array}$ & $\begin{array}{l}\text { Traumatología } \\
(21,2 \%)\end{array}$ & $\begin{array}{l}\text { Medicina } \\
\text { Interma } \\
(14,3 \%)\end{array}$ & $\begin{array}{l}\text { Psiquiatría } \\
(13,7 \%)\end{array}$ & $\begin{array}{l}\text { Pediatŕa } \\
(4 \%)\end{array}$ \\
\hline $\begin{array}{l}\text { Neurología } \\
(9,4 \%)\end{array}$ & $\begin{array}{l}\text { Neurocinugía } \\
(4,5 \%)\end{array}$ & $\begin{array}{l}\text { Neurología } \\
(5,2 \%)\end{array}$ & $\begin{array}{l}\text { Ginecología- } \\
\text { Obstetricia } \\
(7,6 \%)\end{array}$ & $\begin{array}{l}\text { Neurología } \\
(8,8 \%)\end{array}$ & $\begin{array}{l}\text { Cinugía } \\
(10,7 \%)\end{array}$ & $\begin{array}{l}\text { Medicina } \\
\text { Intema } \\
(11,8 \%)\end{array}$ & $\begin{array}{l}\text { Psiquiatńa } \\
(3 \%)\end{array}$ \\
\hline $\begin{array}{l}\text { Psiquiatŕa } \\
(7,5 \%)\end{array}$ & $\begin{array}{l}\text { Traumatología } \\
(4,5 \%)\end{array}$ & $\begin{array}{l}\text { Traumatología } \\
(3,4 \%)\end{array}$ & $\begin{array}{l}\text { Neurología } \\
(7,6 \%)\end{array}$ & $\begin{array}{l}\text { Pediatńa } \\
(5,6 \%)\end{array}$ & $\begin{array}{l}\text { Traumatología } \\
(8,9 \%)\end{array}$ & $\begin{array}{l}\text { Pediatría } \\
(5,9 \%)\end{array}$ & $\begin{array}{l}\text { Traumatología } \\
(2,3 \%)\end{array}$ \\
\hline
\end{tabular}

Para cada año de la carrera se describe las cinco opciones de especialidad más frecuentes dentro de los estudiantes. Para cada alternativa, se detalla el porcentaje de alumnos que la elige, dentro del total de alumnos por año que declara querer especializarse. Dentro de los primeros 4 años de la carrera la medicina interna y sus subespecialidades representa la primera elección. Sin embargo a partir del quinto año, y en especial durante el internado, ésta disminuye, evidenciándose una distribución más homogénea entre las distintas especialidades

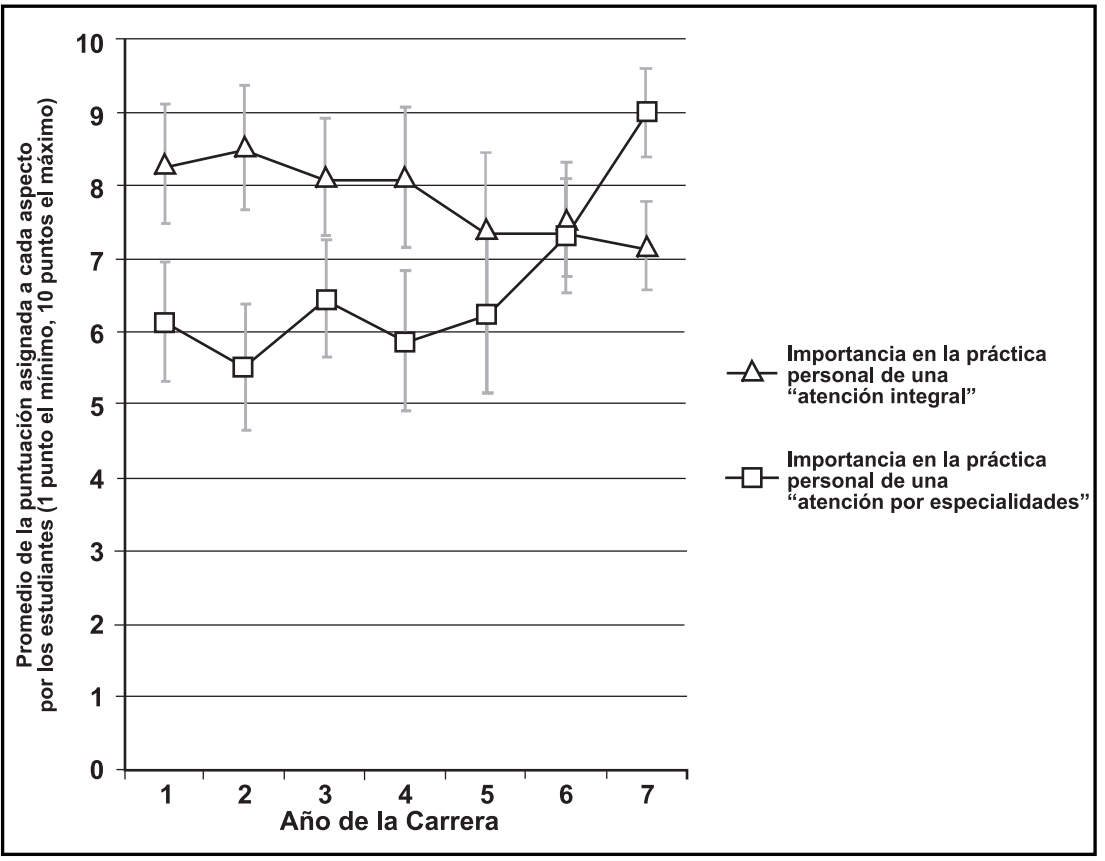

Figura 1. Importancia que los estudiantes asignan a una atención integral y a una atención por especialidades. Se muestran el promedio de las puntuaciones para "atención integral" (triángulos) y para "atención por especialidades" (cuadrados) en cada año de la carrera. Se señalan además la desviación estándar para cada punto. A pesar que en primer año una "atención integral" sea más importante para el estudiante, la tendencia año a año termina por invertir esta preferencia. 


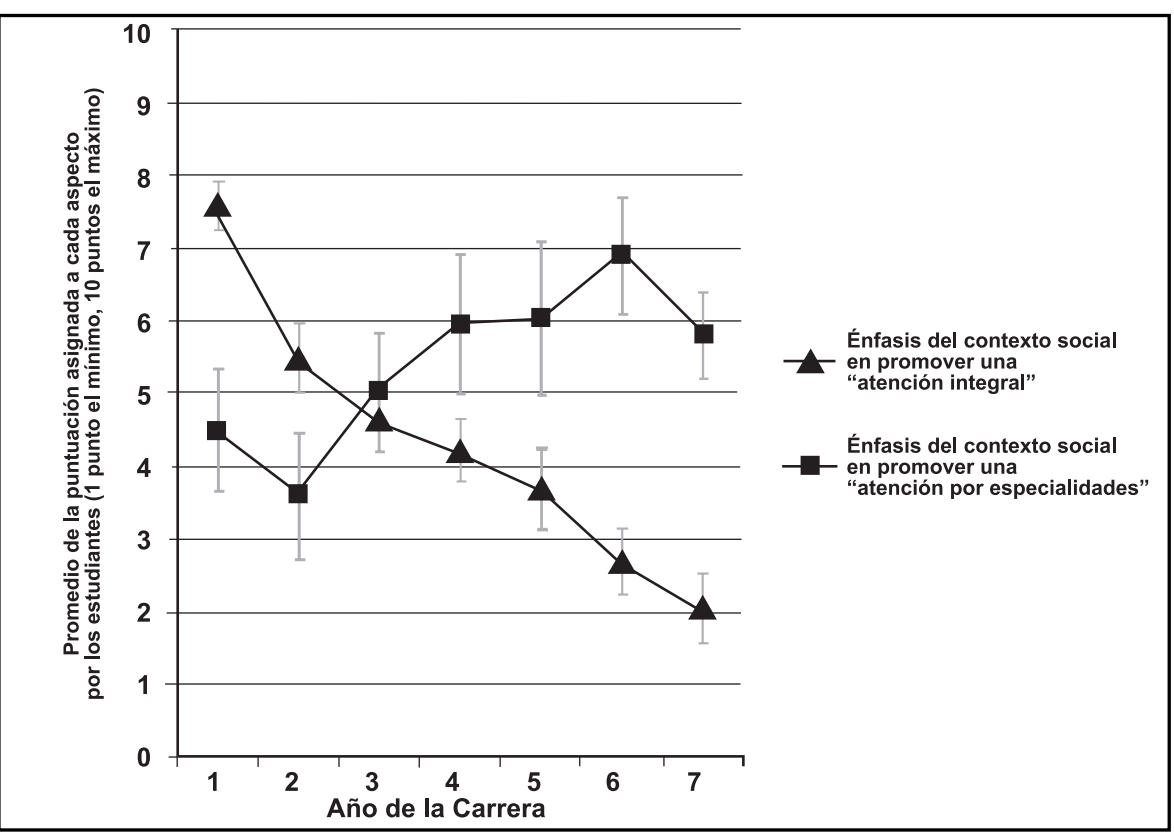

Figura 2. Percepción de los estudiantes sobre el énfasis con que son promovidos en su medio una atención integral y una atención por especialidades. Se muestran el promedio de las puntuaciones para "atención integral" (triángulos) y para "atención por especialidades" (cuadrados) en cada año de la carrera. Se señala además la desviación estándar para cada punto. A pesar que en primer año una "atención integral" es promovida con mayor énfasis, la marcada tendencia año a año, termina por invertir esta preferencia de manera drástica. Desde cuarto año en adelante, los estudiantes perciben a su medio significativamente como más "pro-especialista".

De manera semejante, $10,4 \%$ del cambio observado en la preferencia que los estudiantes muestran hacia una "atención por especialidades", estanía relacionado con el aumento que tiene el énfasis con que se promueve este aspecto $(r=0,104 ; p \varangle 0,05)$.

\section{DisCUSIONES}

Nuestros hallazgos evidencian una fuerte tendencia en los alumnos evaluados a ejercer como ME. Más de la mitad de los estudiantes en el último año de la carrera declara pretender formarse como especialista al egresar.

La proporción MG/ME registrada (32/68, descontando a quienes no saben qué harán al egresar) es semejante a la distribución actual de médicos en el país, y dista mucho de la necesidad nacional. Aunque se aprecia una tendencia leve durante los primeros cinco años hacia el MG, ésta desaparece durante el internado.
Algunos de nuestros resultados llaman la atención sobre posibles factores subyacentes a la elección de ejercer como MG o ME.

Desde el ingreso a la carrera, algunos estudiantes ( $16 \%$ en primer año) manifiestan el deseo de especializarse sin saber qué especialidad específica desarrollar. A partir de quinto año este fenómeno disminuye, quizás al encontrar el alumno una buena alternativa dentro de las especialidades que va conociendo (los cursos de especialidad se dictan a partir de cuarto año14).

Por otro lado, la elección de especialidades específicas varía enormemente durante la carrera. Durante los primeros cuatro años, medicina intema es por gran distancia la opción favorita. A partir de quinto año esta distribución se desordena, cobrando importancia pediatría y psiquiatría en sexto, así como cirugía y ginecología-obstetricia en séptimo. Esto cobra sentido al considerar que hasta cuarto año casi la totalidad de las asignaturas giran en tomo a patología relacionada con medicina intema, en sexto 
se desamollan los intemados de pediatría, psiquiatría y medicina interna, y en séptimo los intemados de cinugía y ginecología-obstetricia ${ }^{14}$. Los estudiantes parecen tender a elegir la especialidad por la que estén pasando en el momento de la encuesta.

Esto revela un patrón, donde aparentemente (y desde el ingreso a la carrera) no importa tanto qué especialidad elegir, mientras se elija una especialidad.

Fuera de la elección explícita por ser ME o MG, los estudiantes de cursos superiores dan mayor importancia a una práctica médica de onientación especialista en desmedro de una integral. El estudiante percibe en el medio que le rodea un énfasis hacia lo especialista que cobra mayor intensidad hacia séptimo año. Este entorno influiría significativamente sobre el médico en formación: desde 10\% del cambio hacia lo especialista observado en los estudiantes estaría en relación con el efecto del entorno universitario-asistencial. Si consideramos la determinación de estos patrones de conducta como fenómenos complejos y multifactoriales, el impacto de esta variable no deja de ser significativo.

Aunque ratificarlo requeriría de herramientas longitudinales y cualitativas de mayor profundidad, una posible interpretación de estos hallazgos apunta a la existencia de un grupo de creencias y tendencias socialmente compartidas, que fomentan una medicina pro-especialista por sobre una orientada a la medicina general.

Dentro de la expeniencia académica, varios son los factores que podńan apoyar esta tendencia social pro-especialista: Contenidos, asignaturas e intemados están estructurados por especialidad; actividades prácticas en atención primaria o salud familiar ocupan una proporción pequeña en la malla curicular, la mayonáa de los docentes son especialistas; y gran parte de las actividades prácticas e intemados se desamollan dentro de hospitales organizados por especialidades ${ }^{6,14,15}$. Además, residentes en formación como especialistas son reconocidos como modelos de conducta y ejemplo a seguir de los estudiantes $^{9}$. Considerando que la formación de actitudes, pautas morales y conductas ocurre esencialmente en actividades prácticas ${ }^{11}$, no es raro que en el internado de sexto y séptimo año (periodo casi exclusivamente práctico y estructurado por especialidades) repunte la intención de ser ME y la tendencia a practicar una medicina onientada a la especialidad.

Fuera del ambiente universitario la figura del especialista pareceńa revestir mayor connotación en téminos de resolutividad clínica, prestigio profesional y valoración económica ${ }^{14}$. Estas representaciones socialmente compartidas del especialista como "ideal al cual aspirar" generan una compleja red de influencias sobre el estudiante, de gran profundidad psicológica.

En comparación, ejercer como MG carece de gran proyección económica o de desarrollo científico-profesional, con una resolutividad limitada y dependiente del especialista ${ }^{5,6}$.

Independientemente de los factores subyacentes, el mundo médico-universitario parece mostrar una marcada tendencia hacia la especialización, la cual empuja al estudiante a especializarse. Escoger ser MG requerina una motivación y claridad excepcional, que permitan al estudiante lidiar con esta tendencia social.

Este fenómeno agravaría el actual déficit de recurso humano médico que presenta la atención primaria 6 , a pesar de ser considerada como la piedra angular de la reforma de salud ${ }^{18}$.

Dentro de posibles soluciones, se ha propuesto aceptar esta tendencia e incorporar especialistas (o residentes en formación de especialidad) a las actividades en consultorios. Esta iniciativa está en curso, aunque reviste numerosas dificultades, como la necesidad de prolongar la duración de los programas de especialidad o la generación de nueva infraestructura y plantas laborales ${ }^{19,20}$.

Otro acercamiento al problema, reside en fomentar en el recién egresado la intención de ejercer como MG, por períodos prolongados y no sólo como un paso intermedio antes de especializarse.

Para ello, resulta necesario generar una verdadera carrera funcionaria para el $\mathrm{MG}$, que considere: aumentar remuneraciones y cargos en atención primaria; generar grados, ascensos y calificaciones definidos a méritos (y no sólo por antigüedad); fomentar la capacidad resolutiva del MG dentro del sistema de salud; y desarmollar estímulos científicos en la forma de cursos de perfeccionamiento, facilidades para asistencia a congresos y reuniones clínicas. Iniciativas en esta línea podnían hacer del MG una altemativa más atractiva para los estudiantes que se encuentran en diversas etapas de revisión y concreción ${ }^{6,20,21}$.

En el ámbito universitario es igualmente importante potenciar en tiempo y calidad docente las actividades en consultorios, atención médica general y salud familiar. El alumno no sólo debe estar mayormente expuesto a las actividades del MG, sino tener la oportunidad de descubrir nuevos atractivos y oportunidad en él. Queremos 
destacar la existencia de importantes avances y cambios curriculares universitarios en este sentido, basadas firmemente en las necesidades nacionales en atención primaria ${ }^{14,16,17}$.

El impacto de estas y otras medidas sobre la motivación del recién egresado a seguir el camino del MG, resta por ser evaluada.

Queremos explicitar varias limitaciones de este estudio. Quizás la más importante es su diseño transversal: No evaluamos el cambio que ocurre en los mismos individuos a lo largo de la carrera, sino a siete grupos distintos de alumnos. Además, cada generación puede tener particularidades que influyan de manera desconocida en estos datos. Por otro lado, la proporción de estudiantes recogida en la muestra respecto al universo alcanza sólo $57 \%$, fenómeno principalmente secundario a la inasisten-

\section{REFERENCIAS}

1. BASCUÑán M. Cambios en la relación médico-paciente y el nivel de satisfacción de los médicos. Rev Méd Chile 2005; 133: 11-6.

2. Horwitz N. El cambio en la práctica médica: Desafíos psicosociales para la profesión. Rev Méd Chile 2004; 132: 768-72.

3. Román O, Acuña M, Señoret M. Disponibilidad de médicos en Chile al año 2004. Rev Méd Chile 2006; 134: 1057-64.

4. Montero J, Muñoz F, Rosselot E, Valdivieso V, Barba R. La medicina general en la organización médica actual. Rev Méd Chile 1996; 124: 1006-14.

5. ROMÁN O, SEÑORET M. Estado actual de las especialidades médicas en Chile: realidad en el sistema público no municipalizado. Rev Méd Chile 2008; 136: 99-106.

6. Román O, Pineda S, Señoret M. Perfil y número de médicos generales que requiere el país. Rev Méd Chile 2007; 135: 1209-15.

7. García-Huidobro D, NúÑEz F, Vargas P, Astudiwos S, HitschFeld M, GeNNERO R ET AL. Expectativas de estudiantes de medicina de pregrado en relación al perfil del médico esperado. Rev Méd Chile 2006; 134: 947-54.

8. Zuger A. Dissatisfaction with medical practice. N Engl J Med 2004; 350: 69-75.

9. Paice E, Heard S, Moss F. How important are role models in making good doctors? BMJ 2002; 325: 707-10.

10. Vincent C, Young M, Phimips A. Why do people sue doctors? A study of patients and relatives taking legal action. Lancet 1994; 343: 1609-13.

11. Roff S, Preece P. Helping medical students to find their moral compasses: ethics teaching for second and third year undergraduates. J Med Ethics 2004; 30: 487-9. cia de muchos alumnos a las actividades académicas donde se implementó el instrumento. Aunque esta proporción de participantes muestra algunas variaciones a lo largo de la carrera no encontramos diferencias estadísticamente significativas entre cursos (salvo una mayor participación en tercer año) y en suma creemos que esto no afecta mayormente la interpretación de nuestros resultados.

Finalmente queremos destacar que nuestros resultados son esencialmente descriptivos y sólo buscan objetivar y caracterizar una tendencia hacia la especialización entre los estudiantes de medicina. Recalcamos que profundizar en las causas de esta tendencia y la búsqueda de intervenciones que ayuden a equilibrar la relación MG/ME en Chile requiere de nuevos estudios en profundidad.

12. Feudtner C, Christakis D, Christakis N. Do clinical Clercks suffer ethical erosion? Student's perception of their ethical environment and personal development. Acad Med 1994; 69: 670-9.

13. Breinbauer H, Fromm G, Araya L Formación valórica del estudiante de medicina: de lo integral a lo especializado. Bol Hosp SJ de Dios 2007; 54: 337-44.

14. Escuela de Medicina, Facultad de Medicina Universidad de Chile [citado 15 julio 2008] Disponible en: www.med.uchile.cl.

15. PARROCHIA E. Reflexiones en tomo a atención de salud y formación médica, con especial énfasis en su concordancia y complementación a través de un intemado ambulatorio de medicina general. Bol Hosp SJ de Dios 2003; 50.

16. Schonhaut L, Milún T, Hanne C. Formación de especialistas en pediatría y su adecuación a la realidad epidemiológica y laboral. Rev Chil Pediatr 2007; 78: 599-606.

17. Rosso P, Velasco N, Moreno R. Reforma del curriculum de pregrado en la Escuela de Medicina de la Pontificia Universidad Católica de Chile: Objetivos, metodología y estado de avance. Rev Méd Chile 1997; 126: 796-807.

18. Diario Oficial Ley 19.937. Modifica el Dt. Ley 2.762 de 1979 con la finalidad de establecer una nueva concepción de la autoridad sanitaria, distintas modalidades de gestión y fortalece la participación ciudadana. Febrero, 2004.

19. IpINZA M. Cómo solucionar el déficit de médicos en los consultorios urbanos municipalizados. Cuan Med Soc 2004; 44: 73-9.

20. Román O. Incorporación de médicos especialistas a la atención primaria cuna paradoja nacional? Rev Méd Chile 2008; 136: 1073-7.

21. Diario Oficial Ley 19.378. Estatuto de Atención Primaria de Salud Municipal. Abril 1995. 